\title{
OPEN Association between ambient air pollution and perceived stress in pregnant women
}

Dirga Kumar Lamichhane ${ }^{1}$, Dal-Young Jung ${ }^{1}$, Yee-Jin Shin ${ }^{2}$, Kyung-Sook Lee ${ }^{3}$, So-Yeon Lee ${ }^{4}$, Kangmo Ahn ${ }^{5,6}$, Kyung Won $\mathrm{Kim}^{7}$, Youn Ho Shin ${ }^{8}$, Dong In Suh ${ }^{9}$, Soo-Jong Hong ${ }^{4 \bowtie} \&$ Hwan-Cheol Kim ${ }^{1 \bowtie}$

Air pollution may influence prenatal maternal stress, but research evidence is scarce. Using data from a prospective cohort study conducted on pregnant women $(n=2153)$, we explored the association between air pollution and perceived stress, which was assessed using the 14-item Perceived Stress Scale (PSS), among pregnant women. Average exposures to particulate matter with an aerodynamic diameter of $<2.5 \mu \mathrm{m}\left(\mathrm{PM}_{2.5}\right)$ or $<10 \mu \mathrm{m}\left(\mathrm{PM}_{10}\right)$, nitrogen dioxide $\left(\mathrm{NO}_{2}\right)$, and ozone $\left(\mathrm{O}_{3}\right)$ for each trimester and the entire pregnancy were estimated at maternal residential addresses using land-use regression models. Linear regression models were applied to estimate associations between PSS scores and exposures to each air pollutant. After adjustment for potential confounders, interquartilerange (IQR) increases in whole pregnancy exposures to $\mathrm{PM}_{2.5}, \mathrm{PM}_{10}$, and $\mathrm{O}_{3}$ in the third trimester were associated with $0.37(95 \%$ confidence interval $[\mathrm{Cl}] 0.01,0.74), 0.54(95 \% \mathrm{Cl} 0.11,0.97)$, and $0.30(95 \% \mathrm{Cl} 0.07,0.54)$ point increases in prenatal PSS scores, respectively. Furthermore, these associations were more evident in women with child-bearing age and a lower level of education. Also, the association between PSS scores and $\mathrm{PM}_{10}$ was stronger in the spring. Our findings support the relationship between air pollution and prenatal maternal stress.

Maternal stress is relatively common during the prenatal period, with reported prevalences ranging from 12 to $92 \%^{1-3}$. Stress perceived by mothers during this period has been associated with adverse maternal (postpartum depression and suicide ${ }^{4}$ ) and fetal outcomes (preterm birth ${ }^{5}$, low birth weight ${ }^{6}$, congenital heart defects ${ }^{7}$, and impaired neonatal cognitive development ${ }^{8}$ ). In addition, higher risks of depression, impulsivity, and cognitive disorders have been reported in adolescence ${ }^{9,10}$ and schizophrenia in adulthood ${ }^{11}$ have been described in the offspring of women that experienced maternal stress during pregnancy. Therefore, identifying risk factors of prenatal maternal stress is a major public health goal.

Psychosocial stress can manifest in many forms, such as perceived stress, anxiety, or depression, and it has been reported that air pollution is associated with perceived stress ${ }^{12}, \operatorname{mood}^{13}$, suicide ${ }^{14}$, symptoms of depression ${ }^{15}$ and anxiety ${ }^{16}$, psychiatric emergency room visits ${ }^{17}$, and mental health disorders, as summarized in a metaanalysis ${ }^{18}$. Perceived stress is widely assessed using the Perceived Stress Scale (PSS; a measure of global stress), in which situations are appraised by individuals as stressful ${ }^{19}$. A longitudinal study of older men reported that exposure to air pollutants, including particulate matter with an aerodynamic diameter of $<2.5 \mu \mathrm{m}\left(\mathrm{PM}_{2.5}\right)$ and nitrogen dioxide $\left(\mathrm{NO}_{2}\right)$, was associated with higher PSS scores, particularly in cold months ${ }^{12}$. However, another study that explored the association between personal exposure to $\mathrm{NO}_{2}$ and PSS in healthy elderly reported nonsignificant results ${ }^{13}$.

\footnotetext{
${ }^{1}$ Department of Occupational and Environmental Medicine, Inha University School of Medicine, Incheon, Republic of Korea. ${ }^{2}$ Department of Psychiatry, Yonsei University College of Medicine, Seoul, Republic of Korea. ${ }^{3}$ Department of Rehabilitation, Hanshin University, Osan, Gyeonggi-do, Republic of Korea. ${ }^{4}$ Department of Pediatrics, Childhood Asthma Atopy Center, Humidifier Disinfectant Health Center, Asan Medical Center, University of Ulsan College of Medicine, Seoul, Republic of Korea. ${ }^{5}$ Department of Pediatrics, Samsung Medical Center, Sungkyunkwan University School of Medicine, Seoul, Republic of Korea. ${ }^{6}$ Environmental Health Center for Atopic Diseases, Samsung Medical Center, Seoul, Republic of Korea. ${ }^{7}$ Department of Pediatrics, Yonsei University College of Medicine, Seoul, Republic of Korea. ${ }^{8}$ Department of Pediatrics, CHA Gangnam Medical Center, CHA University School of Medicine, Seoul, Republic of Korea. ${ }^{9}$ Department of Pediatrics, Seoul National University College of Medicine, Seoul, Republic of Korea. ${ }^{\square}$ email: sjhong@amc.seoul.kr; carpediem@inha.ac.kr
} 
Pregnant women are especially vulnerable to air pollution due to their increased ventilation rate for higher oxygen requirements of the developing fetus and decreased oxygen-binding capability ${ }^{20}$. In addition, the brains of pregnant women are more susceptible to environmental factors due to high metabolic demands ${ }^{16}$, as air pollutants may reach the brain by crossing the blood-brain barrier and trigger neuroinflammation, and subsequent pathological changes ${ }^{21}$. However, few studies have investigated the relationship between air pollution and maternal stress in pregnant women, and the studies undertaken have largely focused on postpartum depression or emotional stress ${ }^{22-25}$; other indicators of psychosocial stress, such as perceived stress, have not been studied in pregnant women. Nonetheless, it has been reported that the prevalence of maternal stress is higher in the prenatal than in the postnatal period ${ }^{26,27}$. Thus, we considered that delineating the nature of the relationship between air pollution and prenatal stress would increase understanding of the mechanism that links air pollution and affective disorders.

This study aimed to explore potential associations between exposure to air pollution during different trimesters and maternal PSS, which was assessed at the 36th week of pregnancy. We hypothesized that higher levels of air pollution would be associated with higher levels of perceived stress among pregnant women.

\section{Results}

Table 1 presents a summary of the statistics of the study population $(n=2153)$. Mean subject age was approximately 33 years, and mean PSS score was 20.2 with a standard deviation of 7.2. $1314(61.0 \%)$ were nulliparous, $2054(95.4 \%)$ had educated to a higher level (college or above), and 32 (1.5\%) were diagnosed with diabetes or hypertension in pregnancy. Most participants had high income $[62.8 \%$ ( $\geq 4$ million won/month)], and the majority were never smokers $(92.3 \%)$.

The distributions of air pollutant concentrations during the entire pregnancy and across the three trimesters are displayed in Table 2. The average concentrations of air pollutants during the first, second, and third trimesters were $27.64,26.68$, and $27.37 \mu \mathrm{g} / \mathrm{m}^{3}$, respectively, for $\mathrm{PM}_{2.5}, 51.17,49.40$, and $50.70 \mu \mathrm{g} / \mathrm{m}^{3}$ for particulate matter with an aerodynamic diameter of $<10 \mu \mathrm{m}\left(\mathrm{PM}_{10}\right), 35.04,34.57$, and $35.30 \mathrm{ppb}$ for $\mathrm{NO}_{2}$, and $44.52,43.45$, and $41.49 \mathrm{ppb}$ for ozone $\left(\mathrm{O}_{3}\right)$. Correlations between pollutants by trimester are provided in Supplementary Table S1. Spearman's correlation coefficients showed a strong relationship between $\mathrm{PM}_{2.5}$ and $\mathrm{PM}_{10}$ during pregnancy (Spearman's correlation coefficient $r=0.82$ to 0.91 ). $\mathrm{PM}_{10}$ showed a moderate positive correlation with $\mathrm{NO}_{2}$ $(r=0.30$ to 0.54$)$, whereas $\mathrm{NO}_{2}$ showed a moderate negative correlation with $\mathrm{O}_{3}(r=-0.46$ to -0.53$)$. Furthermore, levels of air pollution in the second trimester were strongly correlated with the levels for the entire pregnancy ( $r=0.80$ to 0.88 ) (Supplementary Table S2).

Associations between maternal air pollution exposure and PSS score are presented in Table 3. Analyses revealed significant associations between PSS scores and $\mathrm{PM}_{10}$ during the second trimester and entire pregnancy and between PSS scores and $\mathrm{O}_{3}$ during the third trimester before and after adjustment for relevant covariates. In the primary model, we observed significant positive associations between PSS scores and interquartile-range (IQR) increases in $\mathrm{PM}_{2.5}$ and $\mathrm{PM}_{10}$ in the first and second trimesters and entire pregnancy and between PSS scores and $\mathrm{O}_{3}$ in the third trimester. For example, an IQR increase in exposure to $\mathrm{PM}_{2.5}$ and $\mathrm{PM}_{10}$ in whole pregnancy was associated with 0.37 (95\% confidence interval [CI] 0.01, 0.74) and 0.54 (95\% CI 0.11, 0.97) point increases in PSS scores, respectively. Similarly, an IQR increase in exposure to $\mathrm{O}_{3}$ in the third trimester was associated with a $0.30(95 \%$ CI $0.07,0.54)$ point PSS score increase. No significant association was found between $\mathrm{NO}_{2}$ exposure and PSS scores. Regression coefficients were generally higher in the primary model (model 4), which was additionally adjusted for health behaviors and chronic disease variables, compared to the model that was adjusted for only demographic factors (model 2). When air pollutant exposures were categorized into quartiles, highest quartiles of maternal exposure to $\mathrm{PM}_{10}$ during the second trimester and $\mathrm{O}_{3}$ during the third trimester were found to be related to $1.07(95 \%$ CI $0.10,2.05)$ and $1.40(0.48,2.32)$ point increases in PSS scores, respectively (p for trend $=0.021$ and 0.001 , respectively) as compared with those in lowest quartiles (Supplementary Table S3). Subjects in the highest $\mathrm{PM}_{2.5}$ quartile for entire pregnancy had significantly higher PSS scores (p-value $<0.05$ ), whereas those in the highest quartile for $\mathrm{NO}_{2}$ exposure during the first trimester showed only a marginally significant association ( $\mathrm{p}$-value $<0.1$ ) (Supplementary Table S3).

Spline analyses showed that increases in mean $\mathrm{PM}_{10}$ concentration during the second trimester and $\mathrm{O}_{3}$ concentration during the third trimester were associated with higher PSS scores ( $\mathrm{p}$ for overall association $=0.038$ and 0.005 , respectively) (Fig. 1b,d): $\mathrm{PM}_{2.5}$ exposures during the second trimester were only marginally associated with PSS scores ( $p$ for overall association $=0.054$ ) (Fig. 1a). Spline analyses showed linear dose-response relationships between $\mathrm{PM}_{2.5}$ and $\mathrm{PM}_{10}$ concentrations during the second trimester and PSS scores ( $\mathrm{p}$ for nonlinear $=0.735$ and 0.994 , respectively). In contrast, the association between maternal $\mathrm{O}_{3}$ exposure during the third trimester and PSS scores was nonlinear ( $\mathrm{p}$ for nonlinear $=0.030$ ). The overall association between $\mathrm{NO}_{2}$ exposure during the first trimester and PSS scores was insignificant ( $\mathrm{p}$ for overall $=0.376$ ) and did not significantly deviate from linearity ( $\mathrm{p}$ for nonlinear $=0.464)$ (Fig. $1 \mathrm{c})$, which was consistent with estimates obtained using the other models (Supplementary Fig. S1).

Figure 2 shows evidence of effect modification of associations between air pollution and PSS scores by season. Significant interactions were observed between spring and $\mathrm{PM}_{10}$ exposures during the first trimester and entire pregnancy ( $\mathrm{p}$ for interactions $<0.01$ ); positive associations between PSS scores and $\mathrm{PM}_{10}$ were significant in spring, but not in other seasons. Analyses of effect modification by maternal age, parity, education, and income are reported in Supplementary Fig. S2. In age-stratified models, significant associations were observed between $\mathrm{PM}_{10}$ exposures during the first and second trimesters and entire pregnancy and PSS scores and between $\mathrm{O}_{3}$ exposures during the third trimester and PSS scores in pregnant women aged $<35$ years, but not in women $\geq 35$ years old. A significant interaction was observed between $\mathrm{O}_{3}$ exposure during the third trimester and age ( $\mathrm{p}$ for interaction $<0.05$ ), while interactions between $\mathrm{PM}_{10}$ exposure in the second trimester and entire pregnancy and age 


\begin{tabular}{|c|c|}
\hline Characteristics & Mean \pm SD or $n(\%)$ \\
\hline Age (years) & $33.1 \pm 3.6$ \\
\hline \multicolumn{2}{|l|}{ Age group } \\
\hline$<25$ & $7(0.3)$ \\
\hline $25-29$ & $324(15.1)$ \\
\hline $30-34$ & $1121(52.0)$ \\
\hline $35-39$ & $604(28.1)$ \\
\hline$\geq 40$ & $97(4.5)$ \\
\hline \multicolumn{2}{|l|}{ Pre-pregnancy BMI $\left(\mathrm{kg} / \mathrm{m}^{2}\right)$} \\
\hline$<25$ & $2012(93.5)$ \\
\hline$\geq 25$ & $141(6.5)$ \\
\hline \multicolumn{2}{|l|}{ Parity } \\
\hline Nulliparous & $1314(61.0)$ \\
\hline Parous & $661(30.7)$ \\
\hline Missing & $178(8.3)$ \\
\hline \multicolumn{2}{|l|}{ History of smoking } \\
\hline Never & $1988(92.3)$ \\
\hline Ever & $165(7.7)$ \\
\hline \multicolumn{2}{|l|}{ Drinking during pregnancy } \\
\hline No & $1989(92.4)$ \\
\hline Yes & $164(7.6)$ \\
\hline \multicolumn{2}{|l|}{ Occupation } \\
\hline No & $724(33.6)$ \\
\hline Yes & $1429(66.4)$ \\
\hline \multicolumn{2}{|l|}{ Education } \\
\hline Secondary school & $99(4.6)$ \\
\hline College or university & $1579(73.3)$ \\
\hline Graduate school & $475(22.1)$ \\
\hline Gestational age (weeks) & $39.3 \pm 1.1$ \\
\hline \multicolumn{2}{|l|}{ Family income } \\
\hline High ( $\geq 4$ million per month) & $1353(62.8)$ \\
\hline Low (<4 million per month) & $800(37.2)$ \\
\hline \multicolumn{2}{|l|}{ Asthma } \\
\hline No & $2081(96.7)$ \\
\hline Yes & $72(3.3)$ \\
\hline \multicolumn{2}{|l|}{ Thyroid disease } \\
\hline No & $2011(93.4)$ \\
\hline Yes & $142(6.6)$ \\
\hline \multicolumn{2}{|l|}{ Malignant tumor } \\
\hline No & $2123(98.6)$ \\
\hline Yes & $30(1.4)$ \\
\hline \multicolumn{2}{|l|}{ Liver disease } \\
\hline No & $2084(96.8)$ \\
\hline Yes & $69(3.2)$ \\
\hline \multicolumn{2}{|c|}{ Hypertension or diabetes mellitus } \\
\hline No & $2121(98.5)$ \\
\hline Yes & $32(1.5)$ \\
\hline \multicolumn{2}{|l|}{ Season at delivery } \\
\hline Spring & $465(21.6)$ \\
\hline Summer & $432(20.1)$ \\
\hline Autumn & $489(22.7)$ \\
\hline Winter & $590(27.4)$ \\
\hline Missing & $177(8.2)$ \\
\hline PSS score & $20.2 \pm 7.2$ \\
\hline
\end{tabular}

Table 1. Characteristics of participants. BMI body mass index, PSS perceived stress scale, $S D$ standard deviation. $\mathrm{n}=2153$. 


\begin{tabular}{|c|c|c|c|c|c|c|}
\hline Air pollutants & Mean \pm SD & Min & 25th & 50th & 75th & $\operatorname{Max}$ \\
\hline \multicolumn{7}{|l|}{$\mathrm{PM}_{2.5}\left(\mu \mathrm{g} / \mathrm{m}^{3}\right)$} \\
\hline 1st trimester & $27.64 \pm 8.13$ & 11.41 & 21.17 & 27.27 & 32.85 & 57.46 \\
\hline 2nd trimester & $26.68 \pm 7.68$ & 12.18 & 20.53 & 25.96 & 31.52 & 61.99 \\
\hline 3rd trimester & $27.37 \pm 7.88$ & 11.71 & 21.24 & 26.77 & 32.30 & 57.86 \\
\hline Whole pregnancy & $27.19 \pm 5.67$ & 14.83 & 23.21 & 26.05 & 30.09 & 53.73 \\
\hline \multicolumn{7}{|l|}{$\mathrm{PM}_{10}\left(\mu \mathrm{g} / \mathrm{m}^{3}\right)$} \\
\hline 1st trimester & $51.17 \pm 12.40$ & 24.25 & 40.95 & 51.77 & 61.23 & 88.82 \\
\hline 2nd trimester & $49.40 \pm 11.59$ & 25.73 & 39.31 & 49.09 & 58.58 & 81.48 \\
\hline 3rd trimester & $50.70 \pm 12.04$ & 24.48 & 40.78 & 51.19 & 59.91 & 85.67 \\
\hline Whole pregnancy & $50.36 \pm 6.47$ & 34.60 & 45.80 & 50.17 & 54.66 & 73.93 \\
\hline \multicolumn{7}{|l|}{$\mathrm{NO}_{2}(\mathrm{ppb})$} \\
\hline 1st trimester & $35.04 \pm 9.65$ & 2.00 & 29.00 & 35.00 & 41.00 & 76.00 \\
\hline 2nd trimester & $34.57 \pm 9.14$ & 2.00 & 29.00 & 34.00 & 40.00 & 84.00 \\
\hline 3rd trimester & $35.30 \pm 9.36$ & 3.00 & 29.00 & 35.00 & 41.00 & 81.00 \\
\hline Whole pregnancy & $34.94 \pm 7.97$ & 2.00 & 30.00 & 35.00 & 39.00 & 75.00 \\
\hline \multicolumn{7}{|l|}{$\mathrm{O}_{3}(\mathrm{ppb})$} \\
\hline 1st trimester & $44.52 \pm 15.09$ & 5.00 & 31.00 & 44.00 & 57.00 & 88.00 \\
\hline 2nd trimester & $43.45 \pm 14.50$ & 9.00 & 31.00 & 42.00 & 55.00 & 83.00 \\
\hline 3rd trimester & $41.49 \pm 15.00$ & 8.00 & 28.00 & 40.00 & 53.00 & 85.00 \\
\hline Whole pregnancy & $43.22 \pm 7.88$ & 9.00 & 38.00 & 43.00 & 49.00 & 69.00 \\
\hline
\end{tabular}

Table 2. Distributions of maternal air pollution exposure levels for different pregnancy periods. $S D$ standard deviation, $p p b$ parts per billion.

\begin{tabular}{|c|c|c|c|c|c|}
\hline \multirow[b]{2}{*}{ Air pollutants } & \multirow[b]{2}{*}{ Trimester } & Model $1^{\mathrm{a}}$ & Model $2^{b}$ & Model $3^{c}$ & Model $4^{d}$ \\
\hline & & $\beta(95 \% \mathrm{CI})$ & $\beta(95 \% \mathrm{CI})$ & $\beta(95 \% \mathrm{CI})$ & $\beta(95 \% \mathrm{CI})$ \\
\hline \multirow{4}{*}{$\mathrm{PM}_{2.5}$ (IQR: $6.88 \mu \mathrm{g} / \mathrm{m}^{3}$ ) } & First & $0.11(-0.15,0.37)$ & $0.13(-0.12,0.39)$ & $0.27(-0.01,0.54)^{+}$ & $0.29(0.01,0.57)^{*}$ \\
\hline & Second & $0.25(-0.02,0.53)^{+}$ & $0.29(0.02,0.56)^{*}$ & $0.32(0.03,0.61)^{\star}$ & $0.36(0.06,0.65)^{*}$ \\
\hline & Third & $0.04(-0.23,0.30)$ & $0.04(-0.22,0.31)$ & $-0.04(-0.31,0.24)$ & $-0.03(-0.31,0.24)$ \\
\hline & Pregnancy & $0.28(-0.09,0.64)$ & $0.32(-0.05,0.69)^{+}$ & $0.32(-0.05,0.68)^{+}$ & $0.37(0.01,0.74)^{*}$ \\
\hline \multirow{4}{*}{$\mathrm{PM}_{10}$ (IQR: $8.86 \mu \mathrm{g} / \mathrm{m}^{3}$ ) } & First & $0.12(-0.10,0.34)$ & $0.14(-0.08,0.35)$ & $0.26(0.02,0.51)^{\star}$ & $0.29(0.05,0.53)^{\star}$ \\
\hline & Second & $0.25(0.01,0.48)^{*}$ & $0.28(0.05,0.51)^{*}$ & $0.31(0.04,0.59)^{*}$ & $0.36(0.08,0.63)^{*}$ \\
\hline & Third & $0.01(-0.21,0.24)$ & $0.01(-0.21,0.24)$ & $-0.11(-0.35,0.13)$ & $-0.11(-0.35,0.14)$ \\
\hline & Pregnancy & $0.47(0.05,0.88)^{\star}$ & $0.53(0.11,0.94)^{\star}$ & $0.47(0.04,0.90)^{\star}$ & $0.54(0.11,0.97)^{*}$ \\
\hline \multirow{4}{*}{$\mathrm{NO}_{2}$ (IQR: $\left.9.0 \mathrm{ppb}\right)$} & First & $-0.01(-0.30,0.27)$ & $0.06(-0.22,0.35)$ & $0.17(-0.11,0.45)$ & $0.18(-0.10,0.46)$ \\
\hline & Second & $0.02(-0.28,0.32)$ & $0.12(-0.18,0.42)$ & $0.13(-0.18,0.45)$ & $0.13(-0.19,0.45)$ \\
\hline & Third & $-0.24(-0.53,0.05)$ & $-0.17(-0.46,0.12)$ & $-0.15(-0.43,0.14)$ & $-0.16(-0.45,0.12)$ \\
\hline & Pregnancy & $-0.10(-0.44,0.25)$ & $0.01(-0.33,0.36)$ & $0.07(-0.27,0.41)$ & $0.07(-0.27,0.41)$ \\
\hline \multirow{4}{*}{$\mathrm{O}_{3}$ (IQR: $\left.11.0 \mathrm{ppb}\right)$} & First & $-0.10(-0.33,0.12)$ & $-0.13(-0.35,0.09)$ & $-0.13(-0.36,0.09)$ & $-0.15(-0.38,0.08)$ \\
\hline & Second & $0.04(-0.19,0.27)$ & $-0.01(-0.22,0.24)$ & $0.18(-0.11,0.47)$ & $0.19(-0.10,0.48)$ \\
\hline & Third & $0.30(0.07,0.52)^{\star *}$ & $0.30(0.08,0.52)^{\star *}$ & $0.30(0.06,0.54)^{\star}$ & $0.30(0.07,0.54)^{*}$ \\
\hline & Pregnancy & $0.25(-0.18,0.67)$ & $0.18(-0.24,0.61)$ & $0.32(-0.14,0.78)$ & $0.32(-0.14,0.77)$ \\
\hline
\end{tabular}

Table 3. Associations between air pollution exposure (per IQR increase) and PSS scores among pregnant women in single-pollutant models. CI confidence interval, $I Q R$ interquartile range, $p p b$ parts per billion, $P P S$ perceived stress scale. ${ }^{+}$p-value $<0.1 .{ }^{\star}$ p-value $<0.05$. ${ }^{* *}$-value $<0.01$. ${ }^{a}$ Model 1: unadjusted. ${ }^{b}$ Model 2: Model $1+$ maternal age, education, occupation, and income. ${ }^{\mathrm{c}}$ Model 3: Model $2+$ gestational age, maternal smoking, drinking during pregnancy, parity, pre-pregnancy BMI, and season at delivery. ${ }^{\mathrm{d}}$ Model 4: Model $3+$ asthma, thyroid disease, malignant tumors, liver disease, and hypertension or diabetes.

were marginally significant ( $\mathrm{p}$ for interactions $<0.1$ ). Significant positive associations were observed between PSS scores and $\mathrm{PM}_{2.5}$ exposures during the second trimester and entire pregnancy only in women with less than graduate education ( $\mathrm{p}$ for interactions $<0.05$ ). For parity and income, the associations of $\mathrm{PM}_{2.5}, \mathrm{PM}_{10}$, and $\mathrm{O}_{3}$ with PSS were greater for nulliparous women and women with low incomes, but p-values for interaction terms were not significant $(\mathrm{p}>0.05)$. 

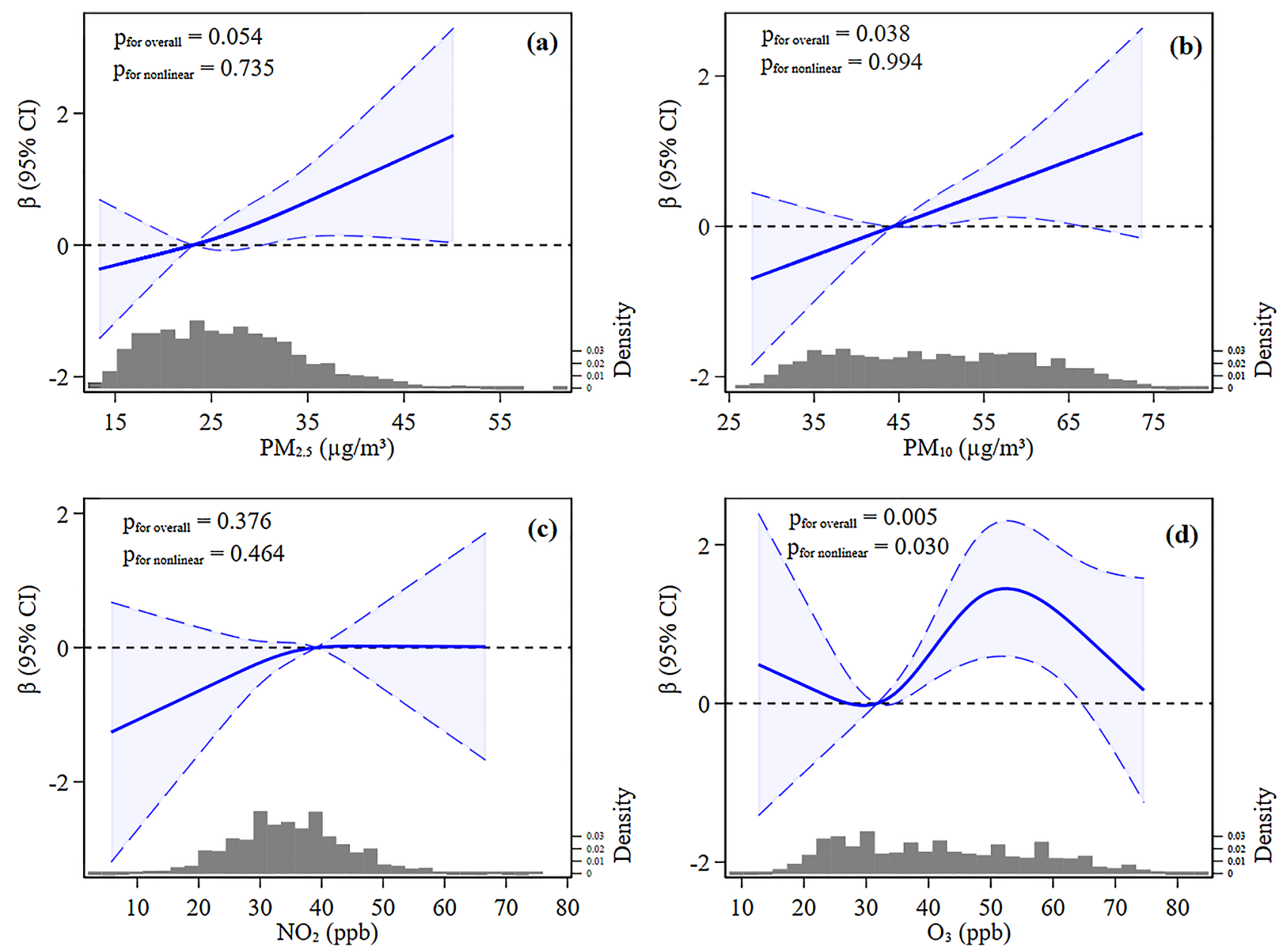

Figure 1. Nonlinear effects of $\mathrm{PM}_{2.5}(\mathbf{a}), \mathrm{PM}_{10}(\mathbf{b}), \mathrm{NO}_{2},(\mathbf{c})$ and $\mathrm{O}_{3}(\mathbf{d})$ on PSS scores. Point estimates (solid line) and 95\% confidence intervals (long dashed lines) were obtained by restricted cubic splines with three knots at the 10th, 50th, and 90th percentiles of $\mathrm{PM}_{2.5}, \mathrm{PM}_{10}$, and $\mathrm{NO}_{2}$ distributions and four knots at the 5th, 35th, 65th, and 95th percentiles of the $\mathrm{O}_{3}$ distribution. $\mathrm{PM}_{2.5}$ and $\mathrm{PM}_{10}$ during the second trimester, $\mathrm{NO}_{2}$ during the first trimester, and $\mathrm{O}_{3}$ during the third trimester were used for spline analyses. Reference $\mathrm{PM}_{2.5}, \mathrm{PM}_{10}, \mathrm{NO}_{2}$, and $\mathrm{O}_{3}$ values for these plots with $\beta$ fixed at 0.0 were $22.4 \mu \mathrm{g} / \mathrm{m}^{3}, 43.9 \mu \mathrm{g} / \mathrm{m}^{3}, 39.0$ parts per billion (ppb), and $31.0 \mathrm{ppb}$, respectively. Models adjusted for maternal age, education, occupation, gestational age, maternal smoking, drinking during pregnancy, parity, pre-pregnancy BMI, season at delivery, income, asthma, thyroid disease, malignant tumors, liver disease, and hypertension or diabetes. Histograms show the distributions of $\mathrm{PM}_{2.5}(\mathbf{a}), \mathrm{PM}_{10}(\mathbf{b}), \mathrm{NO}_{2}(\mathbf{c})$, and $\mathrm{O}_{3}(\mathbf{d})$ exposures.

Sensitivity analyses revealed relationships between maternal exposure to $\mathrm{PM}_{10}$ during the second trimester and entire pregnancy and PSS scores and between maternal exposure to $\mathrm{O}_{3}$ during the third trimester and PSS scores remained significant after adding other pollutants to the primary model (Table 4). A separate analysis was performed using the primary model after excluding mothers with a chronic health condition but this did not materially change estimates of relationships between maternal exposure to air pollution and PSS scores (Supplementary Table S4). Furthermore, similar findings were obtained when these relationships were examined using the multiple imputation technique (Supplementary Table S5). The E-values with lower 95\% CI and relative risks, as determined using the primary model, are provided in Supplementary Table S6. These results indicate that our conclusions are robust to unmeasured confounding bias.

\section{Discussion}

In this prospective cohort study of pregnant women, we investigated the association between exposure to air pollution and maternal PSS scores. We found that maternal exposure to $\mathrm{PM}_{2.5}$ or $\mathrm{PM}_{10}$ during the first and second trimesters and entire pregnancy and exposure to $\mathrm{O}_{3}$ during the third trimester were significantly associated with higher perceived stress during the third trimester, as determined using single-pollutant models, and that associations between $\mathrm{PM}_{10}$ exposure during the second trimester and entire pregnancy and $\mathrm{O}_{3}$ during the third trimester were also significantly associated with PSS scores using multi-pollutant models. In addition, we found evidence of effect modifications of air pollution-perceived stress associations by season, maternal age, and education.

Experimental research provides plausible results for potential associations between air pollution and stress ${ }^{28}$. Yokota et al. showed that exposure to diesel exhaust particles during pregnancy decreased serotonin in mice, 

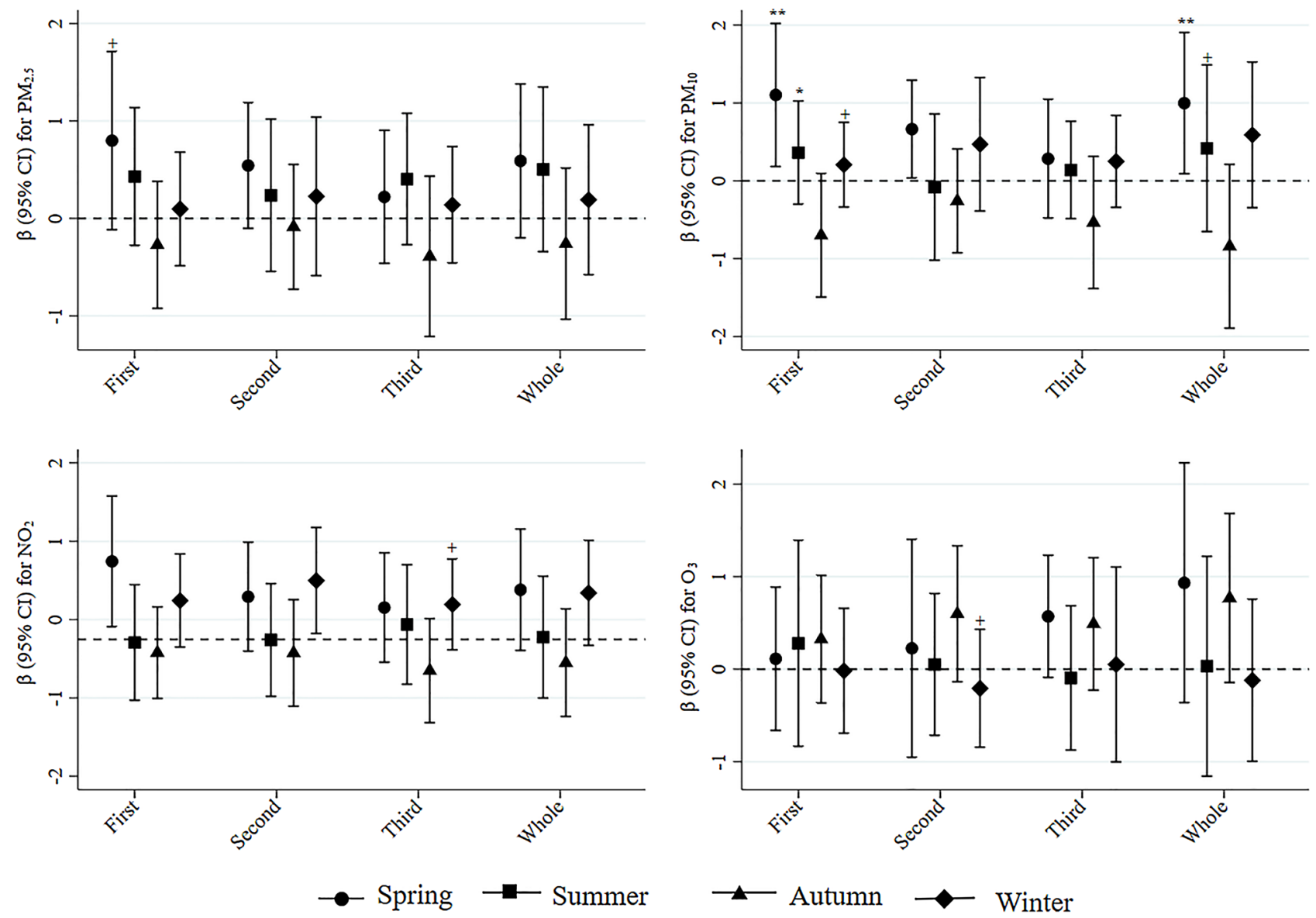

Figure 2. Adjusted differences in PSS scores per IQR increase in air pollutants stratified by season. Analyses were adjusted for maternal age, education, smoking, occupation, drinking during pregnancy, parity, prepregnancy BMI, gestational age, income, asthma, thyroid disease, malignant tumors, liver diseases, and hypertension or diabetes. ${ }^{+} \mathrm{p}$ for interaction $<0.1{ }^{*} \mathrm{p}$ for interaction $<0.05 .{ }^{*} \mathrm{p}$ for interaction $<0.01$.

\begin{tabular}{|c|c|c|c|c|}
\hline \multirow[b]{2}{*}{ Trimester } & $+\mathrm{PM}_{10}$ & $+\mathrm{NO}_{2}$ & $+\mathrm{O}_{3}$ & $+\mathrm{PM}_{10}+\mathrm{NO}_{2}+\mathrm{O}_{3}$ \\
\hline & $\beta(95 \% \text { CI })^{\mathrm{a}}$ & $\beta(95 \% \text { CI })^{a}$ & $\beta(95 \% \mathrm{CI})^{\mathrm{a}}$ & $\beta(95 \% \mathrm{CI})^{\mathrm{a}}$ \\
\hline \multicolumn{5}{|l|}{ First } \\
\hline $\mathrm{PM}_{10}$ & - & $0.29(0.001,0.58)^{\star}$ & $0.28(-0.001,0.56)^{+}$ & $0.28(-0.02,0.59)$ \\
\hline $\mathrm{NO}_{2}$ & $-0.01(-0.34,0.33)$ & - & $0.11(-0.23,0.45)$ & $-0.02(-0.38,0.34)$ \\
\hline $\mathrm{O}_{3}$ & $-0.02(-0.28,0.24)$ & $-0.10(-0.37,0.18)$ & - & $-0.03(-0.31,0.26)$ \\
\hline \multicolumn{5}{|l|}{ Second } \\
\hline $\mathrm{PM}_{10}$ & - & $0.36(0.07,0.65)^{\star}$ & $0.34(0.06,0.61)^{*}$ & $0.31(0.01,0.62)^{*}$ \\
\hline $\mathrm{NO}_{2}$ & $-0.01(-0.35,0.33)$ & - & $0.23(-0.11,0.57)$ & $0.07(-0.31,0.45)$ \\
\hline $\mathrm{O}_{3}$ & $0.13(-0.16,0.43)$ & $0.26(-0.05,0.58)^{+}$ & - & $0.16(-0.17,0.49)$ \\
\hline \multicolumn{5}{|l|}{ Third } \\
\hline $\mathrm{PM}_{10}$ & - & $-0.05(-0.33,0.23)$ & $0.06(-0.22,0.33)$ & $0.05(-0.25,0.34)$ \\
\hline $\mathrm{NO}_{2}$ & $-0.14(-0.47,0.19)$ & - & $0.06(-0.28,0.41)$ & $0.04(-0.32,0.41)$ \\
\hline $\mathrm{O}_{3}$ & $0.33(0.06,0.60)^{\star}$ & $0.33(0.04,0.62)^{*}$ & - & $0.35(0.04,0.65)^{*}$ \\
\hline \multicolumn{5}{|l|}{ Pregnancy } \\
\hline $\mathrm{PM}_{10}$ & - & $0.56(0.11,1.01)^{\star}$ & $0.54(0.11,0.97)^{\star}$ & $0.52(0.07,0.98)^{*}$ \\
\hline $\mathrm{NO}_{2}$ & $-0.07(-0.42,0.29)$ & - & $0.21(-0.17,0.59)$ & $0.06(-0.34,0.46)$ \\
\hline $\mathrm{O}_{3}$ & $0.33(-0.13,0.78)$ & $0.44(-0.07,0.95)^{+}$ & - & $0.36(-0.15,0.88)$ \\
\hline
\end{tabular}

Table 4. Multi-pollutant model of associations between exposure to air pollutants (per IQR increase) and PSS scores among pregnant women. ${ }^{+}$p-value $<0.1 .{ }^{\star}$ p-value $<0.05$. ${ }^{a}$ Multi-pollutant model was further adjusted for the effects of other air pollutants in the same time window using the adjusted single-pollutant model 4 shown in Table 3. 
which can lead to depressive behaviors ${ }^{29}$. Furthermore, epidemiological studies have reported an association between air pollution and specific perceived stress, such as perceived air quality among pedestrians ${ }^{30}$ and nonspecific perceived stress in elderly men ${ }^{12}$. In the present study, we sought to detect changes in non-specific perceived stress in pregnant women. This type of stress indicates that individual perceptions of air quality are unlikely to change due to the range of air pollution levels. In addition, perceived stress is considered an intermediate stage in the association between exposure to air pollution and affective psychiatric disorders. Pregnant women are particularly susceptible to the adverse effects of air pollution. Four studies have so far investigated the relationship between exposure to air pollution during pregnancy and maternal mental health during prenatal and postnatal periods ${ }^{22-25}$. One of these studies reported a link between air pollution and maternal depression during pregnancy ${ }^{22}$, two supported a link between exposure to $\mathrm{PM}_{2.5}$ in pregnancy and postpartum depression ${ }^{23,24}$, and other concluded that exposure to $\mathrm{PM}_{2.5}$ or $\mathrm{NO}_{2}$ from mid to late pregnancy is associated with higher maternal Global Severity-Indices, which indicated higher levels of emotional stress during pregnancy ${ }^{25}$. However, no study has examined the association between exposure to air pollution in different trimesters of pregnancy and prenatal maternal stress.

Stratified analyses showed $\mathrm{PM}_{10}$ exposure had a significant positive effect on PSS scores during spring, but not during other seasons. This finding is consistent with that of a previous Korean study, which reported a stronger association between $\mathrm{PM}_{10}$ exposure and suicide during spring ${ }^{14}$. The average $\mathrm{PM}_{10}$ concentration is relatively high in Seoul during the spring ${ }^{31}$, and thus, pregnant women are probably more likely to perceive air pollution in the spring. However, some studies have reported stronger associations between particulate air pollution and maternal perceived or emotional stress in the cold season ${ }^{12,25}$, which is not in line with our results. This inconsistency is possibly due to seasonal and geographical variations of air pollutant levels and different weather conditions and lifestyles ${ }^{32}$.

Interestingly, we observed a stronger positive association between PSS scores and $\mathrm{PM}_{2.5}$ and $\mathrm{PM}_{10}$ exposures in pregnant women of lower socio-economic status, particularly in women with a lower education level. In Korea, air pollution is known to be spatially associated with lower socio-economic status, which includes considerations of income and educational attainment ${ }^{33,34}$. In addition, individuals with low incomes and less education are more likely to have higher perceived stress levels ${ }^{35}$ and to be more susceptible to the effects of air pollution. Therefore, the combination of more pollution exposure and the influence of indicators of lower socio-economic status may explain the strong associations observed between particulate air pollution and PSS scores among pregnant women in the present study. In addition, women aged $<35$ years showed a significant association between air pollution exposure and perceived stress, whereas older women ( $\geq 35$ years) did not. Young pregnant women are more likely to develop stress-related disorders ${ }^{36}$, which may contribute to the increased vulnerability to air pollution.

Although the mechanisms whereby air pollution affects maternal stress during pregnancy are unclear, we believe our results are biologically plausible. Research has demonstrated that exposure to particulate matter increases inflammation in mouse brains ${ }^{37}$, and chronic brain inflammation is associated with reactive oxygen species formation and oxidative stress, which have been suggested to be biological pathways that lead to mental disorders ${ }^{38,39}$. Higher energy requirements and physiological changes render pregnant women more vulnerable to oxidative stress than the general population ${ }^{40}$. In addition, air pollution has been associated with increased inflammatory markers and altered cytokine production ${ }^{41}$, and maternal exposure to air pollution has also been suggested to cause stress due to activation of the hypothalamic-pituitary-adrenal axis ${ }^{42}$, which is known to be associated with stress and stress-related disorders ${ }^{43,44}$. These findings indicate a possible mediator for the neurotoxic effects of air pollutants on maternal stress.

The major strengths of our study were the use of validated models for assessing the effects of exposure to air pollution and the adjustment for multiple confounders. In addition, the study shows individuals' characteristics have modifying effects on the association between air pollution and PSS scores, which provide insight of susceptibility. Furthermore, multiple sensitivity analysis demonstrated the robustness of our findings, and the use of multi-pollutant models enabled us to conclude that $\mathrm{PM}_{10}$ and $\mathrm{O}_{3}$ exposure are individually associated with higher perceived stress independently of each other. To the best of our knowledge, the present study is the first to investigate the relation between air pollution during pregnancy and the subsequent development of prenatal maternal stress. However, this study has several limitations. First, air pollution estimates were based on maternal residential addresses, and geospatial data on maternal residential history, movement patterns, and time spent indoors and outdoors were unavailable, which may have introduced bias into our model of air pollution exposure, particularly if women with perceived stress have different local mobility patterns. Previous research has indicated maternal mobility during pregnancy is usually limited and generally restricted to residential areas ${ }^{45}$, whereas air pollution tends to be homogenous across local areas. Thus, we believe that movement patterns would have little effect on our exposure estimates. Second, although we controlled for several important potential confounders, our results may have been biased by residual confounding due to unmeasured confounders. For example, it is possible that adjustment for physical activity might have attenuated our results to the null, as inactivity would have reduced exposure to air pollution and been associated with higher levels of perceived stress. Adjustment for socioeconomic status, maternal occupation, and pre-pregnancy BMI may have limited some of the potential confounding caused by physical activity, and therefore, reduced the level of residual confounding. In addition, other factors such as residential noise, meteorologic conditions, and exposure to indoor pollutants might have contributed to confounding or introduced measurement errors that might explain our finding of a positive association between air pollution and PSS scores. To address this issue we evaluated E-values to assess the strength of the relationship and to determine whether a hypothetical unmeasured confounder might have markedly altered our results, but the values obtained suggested they were unlikely to have had a major impact. Third, perceived stress was assessed using self-reports only once during pregnancy, and thus, the responses obtained would not have represented maternal stress status throughout pregnancy. Finally, while the homogenous makeup of the 
Cohort for Childhood Origin of Asthma and allergic diseases (COCOA) study in the Korean population provided excellent internal validity, its generalizability in other populations may be limited.

Nonetheless, the findings of this study have substantial public health implications because perceived stress is a risk factor of affective disorders ${ }^{4}$, cardiovascular disease, and mortality ${ }^{46-48}$. Previous studies have reported that the risk of physical health problems associated with air pollution may be increased by perceived stress ${ }^{49,50}$. However, our findings suggest perceived stress may also be a mediator, which should be taken into consideration. Our findings indicate that air pollution might be a modifiable risk factor of perceived stress during pregnancy. Interventions to improve ambient air quality may reduce women's vulnerability to elevated stress during pregnancy and diminish the risk of poor physical health outcomes associated with stress. In addition, our results highlight the need for perceived stress assessment during pregnancy in areas of high air pollution to prevent exacerbations of affective symptoms during the perinatal period.

\section{Conclusions}

Our findings suggest maternal exposures to $\mathrm{PM}_{2.5}, \mathrm{PM}_{10}$, and $\mathrm{O}_{3}$ are related to higher levels of perceived stress among pregnant women. Pregnant women aged less than 35 years and a lower level of education may be more susceptible to air pollution. In addition, we found that the association between $\mathrm{PM}_{10}$ exposure and PSS scores was stronger in the spring. Future studies are warranted to determine how air pollution exacerbates perceived stress and to assess the effects of perceived stress on associations between air pollution and affective disorders.

\section{Methods}

Study participants. The COCOA was a prospective cohort study of mothers and children conducted at five medical centers and eight public health centers in the Seoul metropolitan area (2007-2015) ${ }^{51}$. A total of 3102 pregnant women of gestational age less than 26 weeks were recruited for the COCOA study. For this analysis, women without PSS data $(n=638)$ were excluded. Women with multiple records $(n=86)$ and missing information on residential address $(n=76)$ were not included in the study. To avoid potential confounding, mothers of preterm babies $(n=75)$ were excluded. Those with missing covariate information $(n=74)$ were also excluded. Overall, 949 women were excluded, and the final sample consisted of 2153 pregnant women. Exclusion criteria details are presented in Supplementary Fig. S3. With the exception of family income, no significant differences were observed those included or excluded (Supplementary Table S7). Data were collected in accordance with relevant guidelines and regulations, and all participants provided written informed consent before being included in the investigation. Ethical approval was provided by the Asan Medical Center (IRB No. 2008-0616), Samsung Medical Center (IRB No. 2009-02-021), Yonsei University (IRB No. 4-2008-0588), the CHA Medical Center (IRB No. 2010-010), and Seoul National University (IRB No. 1401-086-550).

Assessment of prenatal maternal stress. Maternal stress was assessed during the third trimester of pregnancy (36th week of pregnancy) using PSS, which is a 14-item questionnaire intended to assess perception of stress based on responses to seven positively and seven negatively stated questions ${ }^{19,52}$. Responses were rated using a Likert five-point scale, which ranged from "never" (0) to "very often" (4). The scores for the seven positively stated items in the 14-items scale were negated, and total scores were obtained by summing all items. Thus, possible total scores ranged from 0 to 56, and higher scores indicated higher levels of perceived stress. The PSS has been shown to be a valid instrument for the assessment of mental stress among pregnant women $(\text { Cronbach's alpha }=0.88)^{53}$. In the current study, PSS scores were normally distributed and exhibited excellent reliability (Cronbach's alpha $=0.884)$.

Air pollution exposure. The land use regression (LUR) model was used to predict maternal exposures to $\mathrm{PM}_{2.5}, \mathrm{PM}_{10}, \mathrm{NO}_{2}$, and $\mathrm{O}_{3}$. Air pollution data were obtained from the Korean Ministry of the Environment (http://www.airkorea.or.kr/web), which monitored 24-h average concentrations of $\mathrm{PM}_{2.5}, \mathrm{PM}_{10}, \mathrm{NO}_{2}$, and $\mathrm{O}_{3}$ in air daily at a maximum of 40 regulatory sites in Seoul from 2007 to 2015 . Monthly exposures to particulate matter $\left(\mathrm{PM}_{2.5}\right.$ and $\left.\mathrm{PM}_{10}\right), \mathrm{NO}_{2}$, and $\mathrm{O}_{3}$ at maternal residential addresses were estimated using the LUR model, as previously described ${ }^{54,55}$. This model uses several geographical variables, which include traffic indicators, surrounding-land use, topography, and spatial trends, and the final LUR model included lengths of roads, traffic intensities on nearest roads, total heavy-duty traffic on all roads, and a variable that represents spatial trends. Model performance was assessed using leave-one-out cross-validation (LOOCV). The model explained 66-81\% of the variability in measured $\mathrm{PM}_{2.5}, \mathrm{PM}_{10}, \mathrm{NO}_{2}$, and $\mathrm{O}_{3}$ levels, and the predicted values agreed well with measured values, as we previously reported ${ }^{56,57}$. Model-adjusted $\mathrm{R}^{2}$ and LOOCV R $\mathrm{R}^{2}$ values for $\mathrm{PM}_{2.5}, \mathrm{PM}_{10}, \mathrm{NO}_{2}$, and $\mathrm{O}_{3}$ were 0.66 and $0.56,0.69$ and $0.60,0.79$ and 0.73 , and 0.81 and 0.77 , respectively.

To investigate potential critical exposures during pregnancy, we calculated the average concentrations of $\mathrm{PM}_{2.5}, \mathrm{PM}_{10}, \mathrm{NO}_{2}$, and $\mathrm{O}_{3}$ for the three trimesters (1st trimester from 1 to 13 weeks, 2nd trimester from 14 to 27 weeks, and 3rd trimester from 28 weeks to until birth) and entire pregnancy.

Covariates. Potential confounding variables, including maternal age at delivery, maternal education, occupation during pregnancy (yes vs. no), parity (nulliparous vs. parous), pre-pregnancy body mass index (BMI) (kg/ $\mathrm{m}^{2}: \geq 25$ vs. $<25$ ), smoking history (ever vs. never), family income, and drinking during pregnancy (yes vs. no), were ascertained at baseline. Maternal age was divided into five categories $(<25,25-29,30-34,35-39$, and $\geq 40)$, and mother's education was categorized as secondary school, college or university, and graduate school. Family income was dichotomized as high ( $\geq 4$ million Korean won per month) or low $(<4$ million Korean won per month $)^{58}$. The data on gestational age in weeks was obtained from medical records at delivery. We also obtained medical histories according to physicians' diagnoses, including hypertension or diabetes mellitus, asthma, thy- 
roid diseases, malignant tumors, and liver diseases. We used seasons of child birth to investigate seasonal variations in perceived stress during the third trimester. The seasons at delivery were defined as; spring (March to May), summer (June to August), autumn (September to November), and winter (December to February) based on the climate characteristics in South Korea. Previous studies indicate that these variables are related to psychosocial stress and may affect the estimates of associations between exposure to air pollution and perceived stress $^{1,12,59}$.

Statistical analysis. The statistical analysis was performed using STATA version 16.0 (Stata Corporation). Descriptive statistics are presented as mean \pm standard deviations (SDs) or frequencies (\%). Spearman's correlation was used to examine the correlations among air pollutants. Linear regression models were used to assess associations between PSS scores and air pollutant levels averaged over each trimester or whole pregnancy and PSS scores. The regression coefficients were estimated by IQR increases in pollutant concentrations averaged over the pregnancy. Model 1 was used to investigate bivariate associations between PSS scores and air pollutant levels measured during pregnancy. Model 2 was adjusted for age, education, occupation, and income, whereas Model 3 was additionally adjusted for lifestyle and maternal characteristics, which included gestational age, smoking, drinking during pregnancy, parity, pre-pregnancy BMI, and season at delivery. In Model 4, the primary model, we added adjustment for chronic health conditions, which included asthma, thyroid disease, malignant tumors, liver disease, and hypertension or diabetes. In addition to modeling air pollution as continuous variables, it was also modeled as categorical variables by quartiles of air pollution exposure using linear regression for the primary model. We tested for linear trends across quartiles by assigning median air pollutant values to quartile as continuous variables in separate regression models. The results from quartile analysis are reported in Supplementary Table S3.

To explore the potential nonlinear associations between maternal air pollution exposure and PSS scores, we performed restricted cubic spline analysis based on linear regression model ${ }^{60}$. The optimal number of knots for analysis was determined using the Akaike information criterion (AIC). Nonlinearity was assessed by testing the regression coefficients of nonlinear terms, and a p-value of nonlinearity of $<0.05$ was considered to indicate a nonlinear association: the overall association indicated that the regression coefficients of both linear and nonlinear terms of the factor were equal to zero.

In addition, stratified analysis was conducted to examine the effect modification by maternal age, parity, education, income, and season at delivery on the relation between air pollution and perceived stress. To evaluate the significance of effect modification on the multiplicative scale, we included an interaction (product) term between air pollution exposure and each of these five characteristics in our primary model.

Several sensitivity analyses were conducted. First, to test for stability of the effects after controlling for other pollutants, we constructed multi-pollutant models for pregnancy exposure for $\mathrm{PM}_{10}, \mathrm{NO}_{2}$, and $\mathrm{O}_{3}$ but not for $\mathrm{PM}_{2.5}$ due to its high correlation with $\mathrm{PM}_{10}$ (Supplementary Table S1). Second, we reanalyzed the primary model after excluding women with a chronic health condition. Third, we reanalyzed the primary model using multiple imputation technique. Finally, we evaluated the robustness of our results with respect to unmeasured cofounding by calculating the E-values ${ }^{61}$, which indicate the minimum strength of an association that unmeasured confounders must have to explain away associations between exposure (air pollution) and outcome (PSS scores). The E-value of the relative risk estimate for air pollution was calculated using the primary model.

\section{Data availability}

The datasets generated and/or analyzed during the current study are not publicly available, but are available from the corresponding author on reasonable request.

Received: 6 June 2021; Accepted: 17 November 2021

Published online: 06 December 2021

\section{References}

1. Tang, X., Lu, Z., Hu, D. \& Zhong, X. Influencing factors for prenatal stress, anxiety and depression in early pregnancy among women in Chongqing, China. J. Affect. Disord. 253, 292-302 (2019).

2. Kingston, D., Heaman, M., Fell, D., Dzakpasu, S. \& Chalmers, B. Factors associated with perceived stress and stressful life events in pregnant women: Findings from the Canadian maternity experiences survey. Matern. Child Health J. 16, 158-168 (2012).

3. Woods, S. M., Melville, J. L., Guo, Y., Fan, M. Y. \& Gavin, A. Psychosocial stress during pregnancy. Am. J. Obstet. Gynecol. 202(61), e1-61.e7 (2010).

4. Lindahl, V., Pearson, J. L. \& Colpe, L. Prevalence of suicidality during pregnancy and the postpartum. Arch. Womens Ment. Health 8, 77-87 (2005).

5. Staneva, A., Bogossian, F., Pritchard, M. \& Wittkowski, A. The effects of maternal depression, anxiety, and perceived stress during pregnancy on preterm birth: A systematic review. Women Birth 28, 179-193 (2015).

6. Littleton, H. L., Bye, K., Buck, K. \& Amacker, A. Psychosocial stress during pregnancy and perinatal outcomes: A meta-analytic review. J. Psychosom. Obstet. Gynaecol. 31, 219-228 (2010).

7. Rychik, J. et al. Maternal psychological stress after prenatal diagnosis of congenital heart disease. J. Pediatr. 162, 302-307.e1 (2013).

8. Keim, S. A. et al. A prospective study of maternal anxiety, perceived stress, and depressive symptoms in relation to infant cognitive development. Early Hum. Dev. 87, 373-380 (2011).

9. Bergh, B. et al. High antenatal maternal anxiety is related to impulsivity during performance on cognitive tasks in 14 and 15 -yearolds. Neurosci. Biobehav. Rev. 29, 259-269 (2005).

10. Pawlby, S., Hay, D. F., Sharp, D., Waters, C. S. \& O'Keane, V. Antenatal depression predicts depression in adolescent offspring: Prospective longitudinal community-based study. J. Affect. Disord. 113, 236-243 (2009).

11. Mäki, P. et al. Schizophrenia in the offspring of antenatally depressed mothers in the northern Finland 1966 birth cohort: Relationship to family history of psychosis. Am. J. Psychiatry 167, 70-77 (2010). 
12. Mehta, A. J. et al. Associations between air pollution and perceived stress: The Veterans Administration Normative Aging Study. Environ. Health 14, 10 (2015).

13. Nuyts, V., Nawrot, T. S., Scheers, H., Nemery, B. \& Casas, L. Air pollution and self-perceived stress and mood: A one-year panel study of healthy elderly persons. Environ. Res. 177, 108644 (2019).

14. Kim, C. et al. Ambient particulate matter as a risk factor for suicide. Am. J. Psychiatry 167, 1100-1107 (2010).

15. Zhang, Z. et al. Long-term particulate matter exposure and onset of depression in middle-aged men and women. Environ. Health Perspect. 127, 77001 (2019).

16. Pun, V. C., Manjourides, J. \& Suh, H. Association of ambient air pollution with depressive and anxiety symptoms in older adults: Results from the nshap study. Environ. Health Perspect. 125, 342-348 (2017).

17. Buoli, M. et al. Is there a link between air pollution and mental disorders?. Environ. Int. 118, 154-168 (2018).

18. Braithwaite, I., Zhang, S., Kirkbride, J. B., Osborn, D. P. J. \& Hayes, J. F. Air pollution (particulate matter) exposure and associations with depression, anxiety, bipolar, psychosis and suicide risk: A systematic review and meta-analysis. Environ. Health Perspect. 127, 126002 (2019).

19. Cohen, S., Kamarck, T. \& Mermelstein, R. A global measure of perceived stress. J. Health Soc. Behav. 24, 385-396 (1983).

20. Kannan, S., Misra, D. P., Dvonch, J. T. \& Krishnakumar, A. Exposures to airborne particulate matter and adverse perinatal outcomes: A biologically plausible mechanistic framework for exploring potential effect modification by nutrition. Environ. Health Perspect. 114, 1636-1642 (2006).

21. Calderon-Garciduenas, L. et al. Air pollution and your brain: What do you need to know right now. Prim. Health Care Res. Dev. 16, 329-345 (2015).

22. Kanner, J. et al. Chronic exposure to air pollution and risk of mental health disorders complicating pregnancy. Environ. Res. 196, 110937 (2021).

23. Niedzwiecki, M. M. et al. Particulate air pollution exposure during pregnancy and postpartum depression symptoms in women in Mexico City. Environ. Int. 134, 105325 (2020).

24. Sheffield, P. E. et al. Association between particulate air pollution exposure during pregnancy and postpartum maternal psychological functioning. PLoS One 13, e0195267 (2018).

25. Lin, Y. et al. The impacts of air pollution on maternal stress during pregnancy. Sci. Rep. 7, 40956 (2017).

26. Sidebottom, A. C., Hellerstedt, W. L., Harrison, P. A. \& Hennrikus, D. An examination of prenatal and postpartum depressive symptoms among women served by urban community health centers. Arch. Women's Mental Health 17, 27-40 (2014).

27. Dennis, C. L., Falah-Hassani, K. \& Shiri, R. Prevalence of antenatal and postnatal anxiety: Systematic review and meta-analysis. Br. J. Psychiatry 210, 315-323 (2017).

28. Clifford, A., Lang, L., Chen, R., Anstey, K. J. \& Seaton, A. Exposure to air pollution and cognitive functioning across the life course-A systematic literature review. Environ. Res. 147, 383-398 (2016).

29. Yokota, S., Oshio, S., Moriya, N. \& Takeda, K. Social isolation-induced territorial aggression in male offspring is enhanced by exposure to diesel exhaust during pregnancy. PLoS One 11, e0149737 (2016).

30. Pantavou, K., Psiloglou, B., Lykoudis, S., Mavrakis, A. \& Nikolopoulos, G. K. Perceived air quality and particulate matter pollution based on field survey data during a winter period. Int. J. Biometeorol. 62, 2139-2150 (2018).

31. Park, S. K. Seasonal variations of fine particulate matter and mortality rate in Seoul, Korea with a focus on the short-term impact of meteorological extremes on human health. Atmosphere 12, 151 (2021).

32. He, T. et al. Ambient air pollution and years of life lost in Ningbo, China. Sci. Rep. 6, 22485 (2016).

33. Byun, H., Bae, H., Kim, D., Shin, H. \& Yoon, C. Effects of socioeconomic factors and human activities on children's PM10 exposure in inner-city households in Korea. Int. Arch. Occup. Environ. Health 83, 867-878 (2010).

34. Choi, G., Heo, S. \& Lee, J. T. Assessment of environmental injustice in Korea using synthetic air quality index and multiple indicators of socioeconomic status: A cross-sectional study. J. Air Waste Manag. Assoc. 66, 28-37 (2016).

35. Cohen, S. \& Janicki-Deverts, D. Who's stressed? Distributions of psychological stress in the United States in probability samples from 1983, 2006, and 2009. J. Appl. Soc. Psychol. 42, 1320-1334 (2012).

36. Lockwood Estrin, G. et al. Young pregnant women and risk for mental disorders: Findings from an early pregnancy cohort. BJPsych Open 5, e21 (2019).

37. Campbell, A. et al. Particulate matter in polluted air may increase biomarkers of inflammation in mouse brain. Neurotoxicology 26, 133-140 (2005).

38. Fan, S. J. et al. Ambient air pollution and depression: A systematic review with meta-analysis up to 2019. Sci. Total Environ. 701, $134721(2020)$.

39. Ng, F., Berk, M., Dean, O. \& Bush, A. I. Oxidative stress in psychiatric disorders: Evidence base and therapeutic implications. Int. J. Neuropsychopharmacol. 11, 851-876 (2008).

40. Kelly, F. J. Oxidative stress: Its role in air pollution and adverse health effects. Occup. Environ. Med. 60, 612-616 (2003).

41. Fonken, L. K. et al. Air pollution impairs cognition, provokes depressive-like behaviors and alters hippocampal cytokine expression and morphology. Mol. Psychiatry 16, 987-995 (2011).

42. Thomson, E. M., Vladisavljevic, D., Mohottalage, S., Kumarathasan, P. \& Vincent, R. Mapping acute systemic effects of inhaled particulate matter and ozone: Multi-organ gene expression and glucocorticoid activity. Toxicol. Sci. 135, 169-181 (2013).

43. de Rooij, S. R. et al. Associations between DNA methylation of a glucocorticoid receptor promoter and acute stress responses in a large healthy adult population are largely explained by lifestyle and educational differences. Psychoneuroendocrinology 37, 782-788 (2012).

44. Raison, C. L. \& Miller, A. H. When not enough is too much: The role of insufficient glucocorticoid signaling in the pathophysiology of stress-related disorders. Am. J. Psychiatry 160, 1554-1565 (2003).

45. Bell, M. L. \& Belanger, K. Review of research on residential mobility during pregnancy: Consequences for assessment of prenatal environmental exposures. J. Expo. Sci. Environ. Epidemiol. 22, 429-438 (2012).

46. Malik, A. O. et al. Association of perceived stress levels with long-term mortality in patients with peripheral artery disease. JAMA Netw. Open 3, e208741 (2020).

47. Nielsen, N. R., Kristensen, T. S., Schnohr, P. \& Grønbaek, M. Perceived stress and cause-specific mortality among men and women: Results from a prospective cohort study. Am. J. Epidemiol. 168, 481-491 (2008).

48. Redmond, N. et al. Perceived stress is associated with incident coronary heart disease and all-cause mortality in low- but not highincome participants in the Reasons for Geographic and Racial Differences in Stroke study. J. Am. Heart Assoc. 2, e000447 (2013).

49. Chen, E., Schreier, H. M., Strunk, R. C. \& Brauer, M. Chronic traffic-related air pollution and stress interact to predict biologic and clinical outcomes in asthma. Environ. Health Perspect. 116, 970-975 (2008).

50. Clougherty, J. E. et al. Synergistic effects of traffic-related air pollution and exposure to violence on urban asthma etiology. Environ. Health Perspect. 115, 1140-1146 (2007).

51. Yang, H. J. et al. The Cohort for Childhood Origin of Asthma and allergic diseases (COCOA) study: Design, rationale and methods. BMC Pulm. Med. 14, 109 (2014).

52. Park, J. O. \& Seo, Y. S. Validation of the perceived stress scale (PSS) on samples of Korean university students. Korean J. Psychol. 29, 611-629 (2010).

53. Benediktsson, I., McDonald, S. \& Tough, S. Examining the psychometric properties of three standardized screening tools in a pregnant and parenting population. Matern. Child Health J. 21, 253-259 (2017). 
54. Lee, J. Y. et al. Land use regression model for assessing exposure and impacts of air pollutants in school children. J. Korean Soc. Atmos. Environ. 28, 571-580 (2012) (Korean).

55. Yang, S. I. et al. Prenatal PM2.5 exposure and vitamin D-associated early persistent atopic dermatitis via placental methylation. Ann. Allergy Asthma Immunol. 125, 665-673.e1 (2020).

56. Lamichhane, D. K. et al. Lung cancer risk and residential exposure to air pollution: A Korean population-based case-control study. Yonsei Med. J. 58, 1111-1118 (2017).

57. Lamichhane, D. K. et al. Quantile regression analysis of the socioeconomic inequalities in air pollution and birth weight. Environ. Int. 142, 105875 (2020).

58. Cho, Y. M., Kim, C. B., Yeon, K. N., Lee, E. S. \& Kim, K. Trends in the prevalence of childhood asthma in Seoul metropolitan city, Korea: The Seoul atopy. Asthma-friendly school project. J. Prev. Med. Public Health. 51, 275-280 (2018).

59. Park, J. H., Karmaus, W. \& Zhang, H. Prevalence of and risk factors for depressive symptoms in Korean women throughout pregnancy and in postpartum period. Asian Nurs. Res. 9, 219-225 (2015).

60. Desquilbet, L. \& Mariotti, F. Dose-response analyses using restricted cubic spline functions in public health research. Stat. Med. 29, 1037-1057 (2010).

61. VanderWeele, T. J. \& Ding, P. Sensitivity analysis in observational research: Introducing the E-value. Ann. Intern. Med. 167, 268-274 (2017).

\section{Acknowledgements}

The authors would like to express their sincere thanks to the members of the Korea Disease Control and Prevention Agency and COCOA teams.

\section{Author contributions}

All authors, that is, Y.J.S., K.S.L., K.A., K.W.K., Y.H.S., S.J.H., and H.C.K. contributed substantially to the conception and design of the COCOA study. D.Y.J. and H.C.K. were involved in the development or application of exposure modeling. D.K.L. performed the statistical analysis and drafted the manuscript. S.Y.L., D.I.S., and H.C.K. helped supervise the statistical analysis, and S.J.H. and H.C.K. reviewed the manuscript. All authors read and approved the final manuscript.

\section{Funding}

This work was supported by a Research Program funded by the Korea Disease Control and Prevention Agency (Grant nos. 2008-E33030-00, 2009-E33033-00, 2011-E33021-00, 2012-E33012-00, 2013-E51003-00, 2014E51004-00, 2014-E51004-01, 2014-E51004-02, 2017-E67002-00, 2017-E67002-01, 2017-E67002-02, 2019ER671000, and 2019-ER671001).

\section{Competing interests}

The authors declare no competing interests.

\section{Additional information}

Supplementary Information The online version contains supplementary material available at https://doi.org/ 10.1038/s41598-021-02845-4.

Correspondence and requests for materials should be addressed to S.-J.H. or H.-C.K.

Reprints and permissions information is available at www.nature.com/reprints.

Publisher's note Springer Nature remains neutral with regard to jurisdictional claims in published maps and institutional affiliations.

(i) Open Access This article is licensed under a Creative Commons Attribution 4.0 International License, which permits use, sharing, adaptation, distribution and reproduction in any medium or format, as long as you give appropriate credit to the original author(s) and the source, provide a link to the Creative Commons licence, and indicate if changes were made. The images or other third party material in this article are included in the article's Creative Commons licence, unless indicated otherwise in a credit line to the material. If material is not included in the article's Creative Commons licence and your intended use is not permitted by statutory regulation or exceeds the permitted use, you will need to obtain permission directly from the copyright holder. To view a copy of this licence, visit http://creativecommons.org/licenses/by/4.0/.

(c) The Author(s) 2021 